\title{
Preface WISSE 2011
}

Information systems security problems are currently a widespread and growing concern that covers most of the areas of society, such as business, domestic, financial, government, healthcare, and so on. The scientific community is beginning to realize the importance of aligning information systems engineering and security engineering in order to develop more secure information systems.

The First International Workshop on Information Systems Security Engineering (WISSE) expands the vision of the information system engineering from a perspective of security. It aims to identify current research on methods, models, and tools for IS security and to bring together researchers and practitioners who want to discuss key issues in IS security engineering.

This workshop, held in London (United Kingdom) on June 21th, 2011, was organized in conjunction with the 23rd International Conference on Advanced Information Systems Engineering (CAiSE'11).

In order to ensure a high quality workshop, among 12 received papers, the Program Committee selected 4 papers as full papers, thus coming up with an acceptance rate of $33 \%$. The proceedings also includes 2 short papers. The selected research papers for presentation address the following topics:

- Methodologies and models for evaluating IS security aspects,

- Security Architectures and Patterns for Information Systems,

- Secure Information Systems development methodologies,

- Models and approaches for the verification of security properties,

- Ontologies for IS security,

- Integrating functional and security requirements,

- Access and Usage control models,

- Formal methods for modelling security.

Besides these papers, a keynote address deals with modelling and reasoning about security and privacy and how they should be treated under one unified framework. The keynote speaker, Dr. Haris Mouratidis, is widely known for his expertise on this subject and we gratefully acknowledge his contribution.

We wish to thank all the contributors to WISSE'2011, in particular the authors who submitted papers and the members of the Program Committee who carefully reviewed them. Special thanks to Dr. Haris Mouratidis who will honour us by offering the Keynote Speech which we hope you find motivating. We express our gratitude to the CAiSE'11 Workshop Chairs, Oscar Pastor and Camille Salinesi, for their helpful support in preparing the workshop. Finally, we thank our colleagues from the Steering Committee, Nora Cuppens, Jan Jürjens and Luis Enrique Sánchez, for initiating the workshop and contributing to its organization. 


\section{WISSE'2011 Organization}

\section{Workshop co-chairs:}

Nadira Lammari

David G. Rosado

\section{Steering committee}

Nora Cuppens

Jan Jürjens

Luis Enrique Sánchez

\section{Program Committee}

Jacky Akoka

Yudis Asnar

Isabelle Comyn-Wattiau

Frédéric Cuppens

Ernesto Damiani

Sabrina De Capitani di Vimercati

Eduardo B. Fernández

Eduardo Fernández-Medina

Marc Frappier

Paolo Giorgini

Régine Laleau

Jaejoon Lee

Yves Ledru

Javier López

Antonio Maña

Jérémy Milhau

Haralambos Mouratidis

Brajendra Panda

Kouichi Sakurai

Duminda Wijesekera

Louise Yngström

\section{Auxiliary Reviewers}

Christos Kalloniatis

Satoshi Hada

Shareeful Islam

Carlos Blanco

Wook Shin
CEDRIC, CNAM, France

University of Castilla-La Mancha, Spain

LUSSI/SERES Telecom-Bretagne, France Technical University of Dortmund, Germany University of Castilla-La Mancha, Spain

CEDRIC, CNAM, France

Università di Trento, Italy

CEDRIC, CNAM, France

Telecom Bretagne, France

Università degli Studi di Milano, Italy

degli Studi di Milano, Italy

Florida Atlantic University, USA

University of Castilla-La Mancha, Spain

University of Sherbrooke, Québec

University of Trento, Italy

LACL, University of Paris-Est, France

Lancaster University, UK

LIG, University of Grenoble, France

University of Málaga, Spain

University of Malaga, Spain

LACL, University of Paris-Est, France

University of East London, UK

University of Arkansas, USA

Kyushu University, Japan

University George Mason, USA

Stockholm University, Sweden

University of the Aegean. Greece

IBM Research, Japan

Technical University of Munich, Germany

University of Cantabria, Spain

KDDI R\&D Laboratories, Inc., Japan 